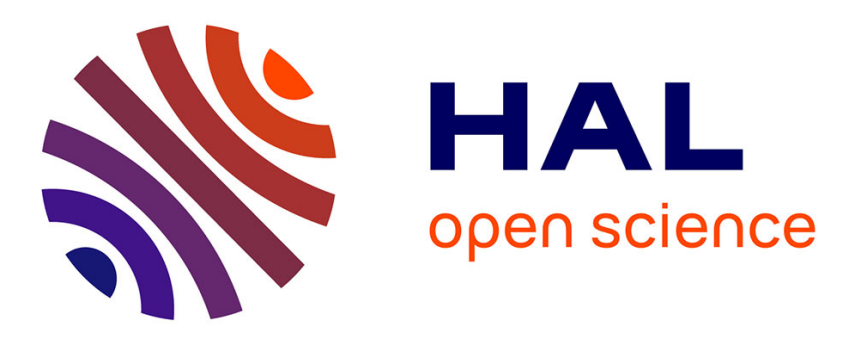

\title{
A robust VAG scheme for a two-phase flow problem in heterogeneous porous media
}

\author{
Konstantin Brenner, Roland Masson, El Houssaine Quenjel
}

\section{To cite this version:}

Konstantin Brenner, Roland Masson, El Houssaine Quenjel. A robust VAG scheme for a two-phase flow problem in heterogeneous porous media. International Conference on Finite Volumes for Complex Applications FVCA 2020, Jun 2020, Bergen, Norway. 10.1007/978-3-030-43651-3_53 . hal-02476745

\section{HAL Id: hal-02476745 \\ https://hal.science/hal-02476745}

Submitted on 12 Feb 2020

HAL is a multi-disciplinary open access archive for the deposit and dissemination of scientific research documents, whether they are published or not. The documents may come from teaching and research institutions in France or abroad, or from public or private research centers.
L'archive ouverte pluridisciplinaire HAL, est destinée au dépôt et à la diffusion de documents scientifiques de niveau recherche, publiés ou non, émanant des établissements d'enseignement et de recherche français ou étrangers, des laboratoires publics ou privés. 


\title{
A robust VAG scheme for a two-phase flow problem in heterogeneous porous media
}

\author{
K. Brenner * , R. Masson * and E.H. Quenjel *
}

February 12, 2020

\begin{abstract}
A positive Vertex Approximate Gradient (VAG) scheme is proposed to discretize the total velocity formulation of two-phase Darcy flow problems in heterogeneous porous media. The discretization is based on the physical variables and allows for multiple rock types with highly contrasted petrophysical and hydrodynamical properties. The numerical experiment shows that, compared to the Phase Potential Upwind (PPU) version of VAG scheme, this new discretization is more robust and efficient in terms of nonlinear convergence
\end{abstract}

Key words : two-phase Darcy flow, finite volume scheme, heterogeneous capillary pressure, VAG scheme MSC : 35K65, 65M08, 65M22, 6T99.

\section{Introduction}

We apply the Vertex Approximate Gradient (VAG) discretization [4 to the two-phase Darcy flow problem written in the total velocity formulation [2]. The choice of the numerical scheme is motivated by the ability of the VAG discretization to properly capture saturation jumps across different rock types. An upwind approximation [7] of the capillary diffusion term with respect to the capillary VAG fluxes is proposed in order to avoid possible undershoots and overshoots of the saturation, that could result from the non monotonicity of the VAG capillary fluxes. The time integration is chosen implicit to avoid severe time step restrictions in high velocity regions. For stability reasons, the pressure and saturation are fully coupled to account for the nonlinear transmission conditions at different rock type interfaces [1. The resulting discretization can be viewed as an extension of the Hybrid Upwind (HU) transport scheme to the VAG discretization. The HU scheme was developed in the framework of the Two-Point Flux Approximation [6] in order to improve the convergence of the nonlinear solver. The authors used only one unknown per rock type interface, typically the saturation or the capillary pressure, whereas our discretization uses both pressure and saturation interface variables. This latter approach allows to accurately represent the capillary barriers. The positive approximation of the transport and capillary diffusion terms ensure a discrete maximum principle on the saturations. Following [3], our choice of the primary unknowns at interface nodes between different rock types is based on a generalization of variable switch techniques which allows to stabilize Newton's method. As shown in the numerical section, this new HU VAG discretization provides faster nonlinear convergence than the VAG discretization based on the Phase Potential Upwinding (PPU) [3].

\footnotetext{
${ }^{*}$ Université Côte d'Azur, Inria, CNRS, Laboratoire J.A. Dieudonné, Team Coffee, Parc Valrose, 06108 Nice cedex 02, France, konstantin.brenner@univ-cotedazur.fr, roland.masson@univ-cotedazur.fr, el-houssaine.quenj@univ-cotedazur.fr
} 


\section{Two-phase Darcy flow model}

Let $t_{f}>0$ and $\Omega$ be a bounded domain of $\mathbb{R}^{d}(d \geq 1)$ such that $\bar{\Omega}=\bigcup_{\mathrm{rt} \in \mathcal{R} \mathcal{T}} \bar{\Omega}_{\mathrm{rt}}$, where $\mathcal{R} \mathcal{T}$ is the set of rock types. The total velocity formulation of the two-phase Darcy flow model reads

$$
\left\{\begin{array}{l}
\phi(\mathbf{x}) \partial_{t} s^{\mathrm{nw}}+\operatorname{div}\left(f^{\mathrm{nw}} \mathbf{V}^{T}-D \Lambda(\mathbf{x})\left(\nabla p_{c}+\left(\rho_{\mathrm{w}}-\rho_{\mathrm{nw}}\right) \mathbf{g}\right)\right)=0, \\
\operatorname{div} \mathbf{V}^{T}=0, \\
\mathbf{V}^{T}=\mathbf{V}^{\mathrm{nw}}+\mathbf{V}^{\mathrm{w}}=-\sum_{\alpha \in\{\mathrm{nw}, \mathrm{w}\}} \eta^{\alpha}\left(\mathbf{x}, s^{\alpha}\right) \Lambda(\mathbf{x})\left(\nabla p^{\alpha}-\rho_{\alpha} \mathbf{g}\right), \\
p_{c}=p^{\mathrm{nw}}-p^{\mathrm{w}} \in \widetilde{P}_{c}\left(\mathbf{x}, s^{\mathrm{nw}}\right), \\
s^{\mathrm{nw}}+s^{\mathrm{w}}=1,
\end{array}\right.
$$

with $\{\mathrm{nw}, \mathrm{w}\}$ denoting the set of non-wetting and wetting phases. In $(2.1), \phi(\mathbf{x})$ denotes the porosity, $s^{\alpha}$ the phase saturation, $\Lambda(\mathbf{x})$ the permeability tensor, $p^{\alpha}$ the phase pressure, $\mathbf{V}^{T}$ the total velocity, and $p_{c}$ the capillary pressure. The phase density $\rho_{\alpha}$ is assumed constant. The gravity acceleration vector is denoted by $\mathbf{g}$ and its norm by $g$. The phase mobility function $\eta^{\alpha}\left(\mathbf{x}, s^{\alpha}\right)$ is defined as the ratio of the relative permeability of the phase over its viscosity. Let $\widetilde{P}_{c}(\mathbf{x}, s)$ denote the monotone graph extension of the capillary pressure function (see [1]). It is assumed that $\widetilde{P}_{c}$ and $\eta^{\alpha}$ are spatially homogeneous in each subdomain $\Omega_{\mathrm{rt}}, \mathrm{rt} \in \mathcal{R} \mathcal{T}$; in addition we assume that the total mobility function $\eta(\mathbf{x}, s)=\eta^{\mathrm{nw}}(\mathbf{x}, s)+\eta^{\mathrm{w}}(\mathbf{x}, 1-s)$ verifies $\eta(\mathbf{x}, s) \geq \eta_{\min }>0$. We then denote by $f^{\text {nw }}$ the non-wetting phase fractional flow function $f^{\mathrm{nw}}(\mathbf{x}, s)=\eta^{\mathrm{nw}}(\mathbf{x}, s) / \eta(\mathbf{x}, s)$, and by $D(\mathbf{x}, s)$ the capillary diffusion coefficient $D(\mathbf{x}, s)=\eta^{\mathrm{w}}(\mathbf{x}, 1-s) f^{\mathrm{nw}}(\mathbf{x}, s)$. The system (2.1) is completed by some initial distribution of $s^{n w}$ and the boundary conditions

$$
\mathbf{V}^{\alpha} \cdot \mathbf{n}=0 \quad \text { on } \Gamma^{N} \times\left(0, t_{f}\right), \quad p^{\alpha}=p_{\text {Dir }}^{\alpha} \quad \text { on } \Gamma^{\text {Dir }} \times\left(0, t_{f}\right) \quad \text { for } \alpha \in\{\mathrm{nw}, \mathrm{w}\},
$$

where $\mathbf{n}$ is the outward normal to $\Gamma^{N}$, and $\partial \Omega=\Gamma^{N} \cup \Gamma^{\text {Dir }}$ with $\left|\Gamma^{\text {Dir }}\right|>0$.

\section{Positive VAG discretization for two-phase Darcy flows}

\subsection{VAG mesh, fluxes and pore volumes}

The VAG discretization considers generalized polyhedral meshes of $\Omega$ [4. Let us briefly recall some notations. Let $\mathcal{M}$ be the set of polyhedral cells of $\Omega$. For each $k \in \mathcal{M}$ we denote the set of nodes of the cell $k$ by $\mathcal{V}_{k}$ and we also denote by $\mathcal{V}=\bigcup_{k \in \mathcal{M}} \mathcal{V}_{k}$ the set of all vertices of the mesh, and by $\mathcal{M}_{\mathbf{s}}$ the subset of cells sharing the node $\mathbf{s} \in \mathcal{V}$. Note that the mesh is supposed to be conforming w.r.t. the partition of $\Omega$ in subdomains $\Omega_{\text {rt }}$, rt $\in \mathcal{R} \mathcal{T}$, and w.r.t. the partition $\left\{\Gamma^{N}, \Gamma^{\text {Dir }}\right\}$ of $\partial \Omega$. We then denote by $\mathcal{V}_{\text {Dir }}$ the set of nodes located at $\bar{\Gamma}^{\text {Dir }}$.

Let $X_{\mathcal{D}}=\left\{v_{k} \in \mathbb{R}, v_{\mathbf{s}} \in \mathbb{R}\right.$, for $\left.k \in \mathcal{M}, \mathbf{s} \in \mathcal{V}\right\}$ be the vector space of degrees of freedom (d.o.f.). The VAG scheme is a control volume scheme in the sense that it results, for each d.o.f. not located at the Dirichlet boundary, in a volume balance equation. The two main ingredients are therefore the conservative fluxes and the pore volumes. For $u_{\mathcal{D}} \in X_{\mathcal{D}}$, the VAG fluxes $F_{k, \mathbf{s}}\left(u_{\mathcal{D}}\right)$ connect the cell $k \in \mathcal{M}$ to its nodes $\mathbf{s} \in \mathcal{V}_{k}$. They can be expressed as $F_{k, \mathbf{s}}\left(u_{\mathcal{D}}\right)=\sum_{\mathbf{s}^{\prime} \in \mathcal{V}_{k}} \mathbb{T}_{k}^{\mathbf{s}, \mathbf{s}^{\prime}}\left(u_{k}-u_{\mathbf{s}^{\prime}}\right)$, with $\left(\mathbb{T}_{k}^{\mathbf{s}, \mathbf{s}^{\prime}}\right)_{\mathbf{s}, \mathbf{s}^{\prime} \in \mathcal{V}_{k}}$ a symmetric positive definite matrix obtained from the $\mathbb{P}_{1}$ finite element subspace defined on a tetrahedral submesh of $\mathcal{M}$.

As described in [3], the portions $\phi_{k, \mathbf{s}}$ of each cell's pore volume $\int_{k} \phi(\mathbf{x}) d \mathbf{x}$ is distributed to its nodes $\mathbf{s} \in \mathcal{V}_{k} \backslash \mathcal{V}_{\text {Dir }}$. We then define $\phi_{k}=\int_{k} \phi(\mathbf{x}) d \mathbf{x}-\sum_{\mathbf{s} \in \mathcal{V}_{k} \backslash \mathcal{V}_{\text {Dir }}} \phi_{k, \mathbf{s}}$ as the remaining cell pore volume. 


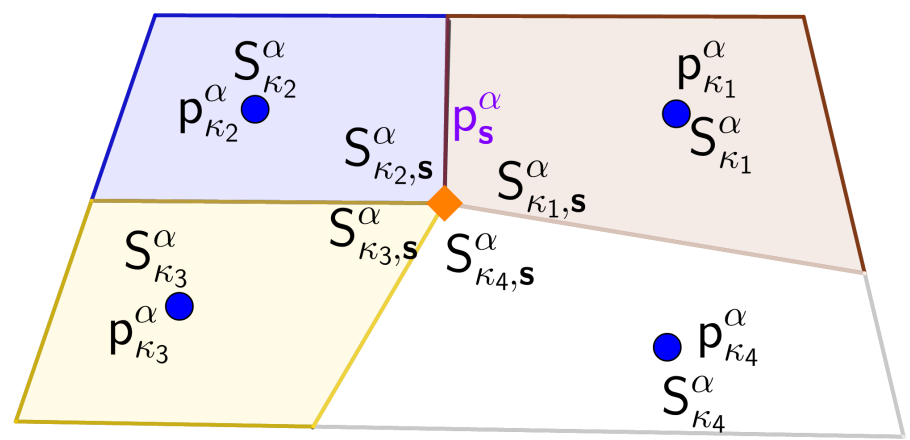

Figure 1: Phase pressure and saturation discrete unknowns in the four cells sharing the vertex s. Each color represents a possible different rock type.

\subsection{Choice of the primary unknowns}

We recall that the mesh is conforming w.r.t. the rock type subdomains, therefore a single rock type $\mathrm{rt}_{k}$ is assigned to each cell $k \in \mathcal{M}$. We denote by $\chi_{\mathbf{s}}=\left\{\mathrm{rt}_{k}, k \in \mathcal{M}_{\mathbf{s}}\right\}$ the set of rock types surrounding the node $\mathbf{s} \in \mathcal{V}$, and we set $\chi_{k}=\left\{\mathrm{rt}_{k}\right\}$ for all $k \in \mathcal{M}$.

The choice of the primary variables follows the variable switching strategy introduced in [3]. We use the pressure of the non-wetting phase as the first primary variable for all d.o.f.; then for the cells and the nodal d.o.f. associated with a single rock type the second primary unknown is the saturation, while for the nodes $\mathbf{s}$ located at rock type interfaces we invoke the variable switching based on a parametrization of $\widetilde{P}_{c, \mathrm{rt}}$, rt $\in \chi_{\mathbf{s}}$. For such nodes we construct a set of non-decreasing continuous functions $P_{c, \chi_{\mathbf{s}}}(\tau)$ and $\left.\left(S_{\chi_{\mathrm{s}}, \mathrm{rt}}^{\mathrm{nw}}(\tau)\right)\right)_{\mathrm{rt} \in \chi_{\mathrm{s}}}$ defined for $\tau \in[0,1]$ and satisfying $P_{c, \chi_{\mathbf{s}}}(\tau) \in \widetilde{P}_{c, \mathrm{rt}}\left(S_{\chi_{\mathbf{s}}, \mathrm{rt}}^{\mathrm{nw}}(\tau)\right)$ for all $\mathrm{rt} \in \chi_{\mathbf{s}}$ and $\tau \in[0,1]$; in addition we require $P_{c, \chi_{\mathbf{s}}}(\tau)+\sum_{\mathrm{rt} \in \chi_{\mathrm{s}}} S_{\chi_{\mathbf{s}}, \mathrm{rt}}^{\mathrm{nw}}(\tau)$ to be strictly increasing. We note that this parametrization allows to deal both with a vanishing capillary diffusion and the capillary barriers. The functions $\left.\tau \mapsto P_{c, \chi_{\mathbf{s}}}(\tau),\left(S_{\chi_{\mathbf{s}}, \mathrm{rt}}^{\mathrm{nw}}(\tau)\right)\right)_{\mathrm{rt} \in \chi_{\mathbf{s}}}$ have to be chosen carefully in order to improve the nonlinear solver and we refer to [3] for a detailed discussion. In order to unify the notations, for the cells or the nodes associated with a single rock type, we introduce a trivial parametrization defined by $S_{\chi, \mathrm{rt}}^{\mathrm{nw}}(\tau)=\tau$ for $\chi$ reduced to a single rock type.

Given the primary unknowns $p_{\mathcal{D}}^{\mathrm{nw}}=\left(p_{\nu}^{\mathrm{nw}}\right)_{\nu \in \mathcal{M} \cup \mathcal{V}}$ and $\tau_{\mathcal{D}}=\left(\tau_{\nu}\right)_{\nu \in \mathcal{M} \cup \mathcal{V}}$, we define

$$
\left\{\begin{aligned}
p_{c, \mathcal{D}} & =\left(p_{c, \nu}\right)_{\nu \in \mathcal{M} \cup \mathcal{V},}, & & \text { with } p_{c, \nu}=P_{c, \chi_{\nu}}\left(\tau_{\nu}\right) \\
p_{\mathcal{D}}^{\mathrm{w}} & =\left(p_{\nu}^{\mathrm{w}}\right)_{\nu \in \mathcal{M} \cup \mathcal{V},}, & & \text { with } p_{\nu}^{\mathrm{w}}=p_{\nu}^{\mathrm{nw}}-p_{c, \nu} \\
\Phi_{\mathcal{D}}^{\alpha} & =p_{\mathcal{D}}^{\alpha}+\rho_{\alpha} g Z_{\mathcal{D}}, & & \text { with } Z_{\mathcal{D}}=\left(z_{\nu}\right)_{\nu \in \mathcal{M} \cup \mathcal{V}} \\
s_{k}^{\alpha} & =S_{\chi_{k}, \mathrm{rt}_{k}}^{\alpha}\left(\tau_{k}\right), & & k \in \mathcal{M} \\
s_{k, \mathbf{s}}^{\alpha} & =S_{\chi_{\mathbf{s}}^{\alpha}, \mathrm{rt}_{k}}\left(\tau_{\mathbf{s}}\right), & & \mathbf{s} \in \mathcal{V}, k \in \mathcal{M}_{\mathbf{s}}
\end{aligned}\right.
$$

To sum up, as exhibited in Figure 1, the discrete phase pressure is single-valued for all d.o.f. while the saturation is single-valued for all cells (and single rock type nodes) and multi-valued at any node sharing multiple rock types.

\subsection{Hybrid Upwinding (HU) VAG scheme for the diphasic model}

The gravity and capillary gradient fluxes are defined by

$$
G_{k, \mathbf{s}}=\left(\rho_{\mathrm{nw}}-\rho_{\mathrm{w}}\right) g F_{k, \mathbf{s}}\left(Z_{\mathcal{D}}\right), \quad C_{k, \mathbf{s}}=F_{k, \mathbf{s}}\left(p_{c, \mathcal{D}}\right) .
$$


Let us introduce the total velocity fluxes $V_{k, \mathbf{s}}^{T}=V_{k, \mathbf{s}}^{T}\left(p_{\mathcal{D}}^{\mathrm{nw}}, \tau_{\mathcal{D}}\right)$, for all $k \in \mathcal{M}, \mathbf{s} \in \mathcal{V}_{k}$, as well as the following discrete phase saturation functions at each d.o.f.

$$
\gamma_{k}^{\alpha}(\tau)=S_{\chi_{k}, \mathrm{rt}_{k}}^{\alpha}(\tau), \quad k \in \mathcal{M}, \quad \gamma_{\mathbf{s}}^{\alpha}(\tau)=\sum_{k \in \mathcal{M}_{\mathbf{s}}} \frac{\phi_{k, \mathbf{s}}}{\phi_{\mathbf{s}}} S_{\chi_{\mathbf{s}}, \mathrm{rt}_{k}}^{\alpha}(\tau), \quad \mathbf{s} \in \mathcal{V} \backslash \mathcal{V}_{\mathrm{Dir}}
$$

with $\phi_{\mathbf{s}}=\sum_{k \in \mathcal{M}_{\mathbf{s}}} \phi_{k, \mathbf{s}}$. For $N \in \mathbb{N}^{*}$, we consider the time subdivision $t^{0}=0<t^{1}<\cdots<t^{n-1}<t^{n} \cdots<$ $t^{N}=t_{f}$ of $\left[0, t_{f}\right]$. We denote the time steps by $\Delta t^{n}=t^{n}-t^{n-1}$ for all $n=1, \cdots, N$. In the sequel, we omit the time superscript $t^{n}$ in the flux terms. Then, for a given $\tau_{\mathcal{D}}^{0} \in[0,1]^{\mathcal{M} \cup \mathcal{V}}$, the scheme consists in finding $\left(p_{\mathcal{D}}^{\mathrm{nw}, n}, \tau_{\mathcal{D}}^{n}\right)$, solutions of the following system of equations for $\alpha \in\{\mathrm{nw}, \mathrm{w}\}$ :

$$
\begin{cases}\frac{\phi_{k}}{\Delta t^{n}}\left(\gamma_{k}^{\alpha}\left(\tau_{k}^{n}\right)-\gamma_{k}^{\alpha}\left(\tau_{k}^{n-1}\right)\right) & \\ \quad+\sum_{\mathbf{s} \in \mathcal{V}_{k}} f_{k, \mathbf{s}}^{\mathrm{nw}} V_{k, \mathbf{s}}^{T}+D_{k, \mathbf{s}}^{\mathrm{cap}} C_{k, \mathbf{s}}+D_{k, \mathbf{s}}^{\mathrm{g}} G_{k, \mathbf{s}}=0, & k \in \mathcal{M}, \\ \frac{\phi_{\mathbf{s}}}{\Delta t^{n}}\left(\gamma_{\mathbf{s}}^{\alpha}\left(\tau_{\mathbf{s}}^{n}\right)-\gamma_{\mathbf{s}}^{\alpha}\left(\tau_{\mathbf{s}}^{n-1}\right)\right) & \\ \quad-\sum_{k \in \mathcal{M}_{\mathbf{s}}} f_{k, \mathbf{s}}^{\mathrm{nw}} V_{k, \mathbf{s}}^{T}+D_{k, \mathbf{s}}^{\mathrm{cap}} C_{k, \mathbf{s}}+D_{k, \mathbf{s}}^{\mathrm{g}} G_{k, \mathbf{s}}=0, & \mathbf{s} \in \mathcal{V} \backslash \mathcal{V}_{\text {Dir }}, \\ p_{\mathbf{s}}^{\mathrm{nw}, n}=p_{\mathbf{s}, \text { Dir }}^{\mathrm{nw}}, \quad \tau_{\mathbf{s}}^{n}=\tau_{\mathbf{s}, \text { Dir }}, & \mathbf{s} \in \mathcal{V}_{\text {Dir }}\end{cases}
$$

Summing the conservation equations over the phases $\alpha \in\{\mathrm{nw}, \mathrm{w}\}$ provides the discrete divergence-free property of the total velocity fluxes

$$
\sum_{\mathbf{s} \in \mathcal{V}_{k}} V_{k, \mathbf{s}}^{T}=0 \text { for all } k \in \mathcal{M}, \quad \sum_{k \in \mathcal{M}_{\mathbf{s}}} V_{k, \mathbf{s}}^{T}=0 \text { for all } \mathbf{s} \in \mathcal{V} \backslash \mathcal{V}_{\text {Dir }}
$$

Fractional flow term: We first specify two expressions of $V_{k, \mathbf{s}}^{T}$ using the upwind mobilities

$$
V_{k, \mathbf{s}}^{T}=\sum_{\alpha \in\{\mathrm{nw}, \mathrm{w}\}} \eta_{\mathrm{rt}_{k}}^{\alpha}\left(s_{k}^{\alpha}\right) F_{k, \mathbf{s}}\left(\Phi_{\mathcal{D}}^{\alpha}\right)^{+}-\eta_{\mathrm{rt}_{k}}^{\alpha}\left(s_{k, \mathbf{s}}^{\alpha}\right) F_{k, \mathbf{s}}\left(\Phi_{\mathcal{D}}^{\alpha}\right)^{-}
$$

with $x^{ \pm}=\max ( \pm x, 0)$, or alternatively, using the cell mobilities

$$
V_{k, \mathbf{s}}^{T}=\sum_{\alpha \in\{\mathrm{nw}, \mathrm{w}\}} \eta_{\mathrm{rt}_{k}}^{\alpha}\left(s_{k}^{\alpha}\right) F_{k, \mathbf{s}}\left(\Phi_{\mathcal{D}}^{\alpha}\right)
$$

This last choice is expected to be more stable than (3.3). Then, the fractional flow fluxes are defined by

$$
f_{k, \mathbf{s}}^{\mathrm{nw}} V_{k, \mathbf{s}}^{T}=f_{k}^{\mathrm{nw}}\left(s_{k}^{\mathrm{nw}}\right)\left(V_{k, \mathbf{s}}^{T}\right)^{+}-f_{k}^{\mathrm{nw}}\left(s_{k, \mathbf{s}}^{\mathrm{nw}}\right)\left(V_{k, \mathbf{s}}^{T}\right)^{-} .
$$

Capillary term: The capillary gradient flux is not monotone. To tackle this issue, we perform a positive correction as follows

$$
D_{k, \mathbf{s}}^{\mathrm{cap}} C_{k, \mathbf{s}}=\frac{\eta_{\mathrm{rt}_{k}}^{\mathrm{nw}}\left(s_{k}^{\mathrm{nw}}\right) \eta_{\mathrm{rt}_{k}}^{\mathrm{w}}\left(s_{k, \mathbf{s}}^{\mathrm{w}}\right)}{\eta_{\mathrm{rt}_{k}}\left(s_{k}^{\mathrm{nw}, n-1}\right)} C_{k, \mathbf{s}}^{+}-\frac{\eta_{\mathrm{rt}_{k}}^{\mathrm{nw}}\left(s_{k, \mathbf{s}}^{\mathrm{nw}}\right) \eta_{\mathrm{rt}_{k}}^{\mathrm{w}}\left(s_{k}^{\mathrm{w}}\right)}{\eta_{\mathrm{rt}_{k}}\left(s_{k}^{\mathrm{nw}, n-1}\right)} C_{k, \mathbf{s}}^{-} .
$$

Gravity term: The gravity contribution is defined similarly as in (3.5).

Remark 3.1. Note that the explicit approximation $\eta_{\mathrm{rt}_{k}}\left(s_{k}^{\mathrm{nw}, n-1}\right)$ is considered in (3.5). This choice improves the nonlinear convergence without involving any restriction on the time step.

We now state two properties of the scheme (3.1)-(3.2) that can be proved assuming that the capillary functions are bounded. We refer to [5] for the proof.

Proposition 3.1. Let $\tau_{\mathcal{D}}^{0} \in[0,1]^{\mathcal{M} \cup \mathcal{V}}$, then, every solution $\left(p_{\mathcal{D}}^{\mathrm{nw}, n}, \tau_{\mathcal{D}}^{n}\right)$, to the system (3.1) $-(3.2)$ ensures $\tau_{\mathcal{D}}^{n} \in[0,1]^{\mathcal{M} \cup \mathcal{V}}$, meaning that the saturations and the capillary pressure satisfy the physical bounds.

Proposition 3.2. Let $\tau_{\mathcal{D}}^{0} \in[0,1]^{\mathcal{M} \cup \mathcal{V}}$ and let us assume that the total velocity fluxes are given. Then, the saturation equation (3.1) admits a solution $\tau_{\mathcal{D}}^{n}$. 


\section{Numerical results}

We test the ability of the introduced HU VAG scheme to simulate oil migration in a fractured reservoir. It is compared to the PPU VAG version [3] which upwinds the phase mobility w.r.t the phase potential. The domain is $\Omega=(0,100 \mathrm{~m})^{3}$ containing a network of planar fractures of aperture $1 \mathrm{~cm}$ (see Figure 2). The matrix porosity is set to $\phi_{m}=0.2$ and the fracture porosity to $\phi_{f}=0.4$. The matrix permeability is isotropic and set to $\Lambda_{m}=10^{-16} \mathrm{~m}^{2}$ while the fracture tangential permeability is set to $\Lambda_{f}=10^{-10} \mathrm{~m}^{2}$. The oil density is $\rho_{\mathrm{nw}}=700 \mathrm{Kg} / \mathrm{m}^{3}$ and the water density is $\rho_{\mathrm{w}}=1000 \mathrm{Kg} / \mathrm{m}^{3}$. The oil viscosity is set to $\mu^{\mathrm{nw}}=0.005 \mathrm{~Pa}$.s and the water viscosity is $\mu^{\mathrm{w}}=0.001 \mathrm{~Pa}$.s. The fracture and matrix relative permeabilities are given by $k_{r, f}^{\alpha}\left(s^{\alpha}\right)=\left(s^{\alpha}\right)^{1.2}$ and $k_{r, m}^{\alpha}\left(s^{\alpha}\right)=\left(s^{\alpha}\right)^{2}, \alpha=$ $n w, w$. The capillary pressure is $P_{c, m}\left(s^{n w}\right)=-10^{4} \log \left(1-s^{n w}\right)$ in the matrix and $P_{c, f}\left(s^{n w}\right)=-10^{3} \log \left(1-s^{n w}\right)$ in the fracture network. The domain is meshed using a tetrahedral mesh with 47670 cells and 1670 fracture faces. The reservoir is initially saturated with water. Dirichlet boundary conditions are imposed at the top boundary with a wetting phase pressure of $1 \mathrm{MPa}$ and $s^{w}=1$, while at the lower boundary, intersected by the fracture network, we impose $p^{w}=4 \mathrm{MPa}$ and the capillary pressure resulting in the matrix saturation $s^{n w}$ equal to 0.9. The lateral boundaries are assumed impervious and the final time is fixed to $t_{f}=3600$ days. The time stepping is defined by $\Delta t^{1}=\Delta t_{\text {init }}$ and for all $n \geq 1$ by $\Delta t^{n+1}=\max \left(\Delta t_{\max }, 1.2 \Delta t^{n}\right)$, in case of a successful time step $\Delta t^{n}$, and $\Delta t^{n+1}=0.5 \Delta t^{n}$, if Newton's method fails to converge in 25 iterations. We have used the values $\Delta t_{\text {init }}=0.01$ and $\Delta t_{\max }=100$ days.

We display in Figures 36 and Table 1 the results obtained for the PPU and HU VAG discretizations corresponding to the

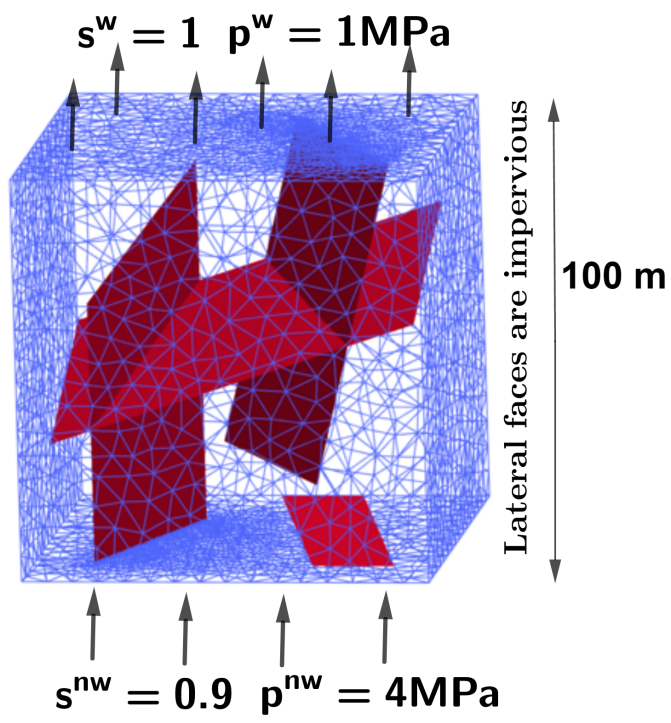

Figure 2: Test case configuration. upwind mobility (3.3) labeled with HU-EtaKs and the centered one (3.4) labeled with HU-EtaK. Figure 3 shows a very good match between the PPU and HU-ETaKs schemes while Figure 4 and 5 exhibit small differences in the matrix between both HU VAG schemes.

The increased robustness of the nonlinear convergence provided by the HU VAG schemes compared with the PPU version is clearly seen in this Table 1 as well as in Figure 6.

\begin{tabular}{lccccc}
\hline Scheme & $N_{\Delta t}$ & $N_{\text {chop }}$ & $N_{\text {Newton }}$ & $N_{\text {GMRes }}$ & CPU $(\mathrm{s})$ \\
\hline PPU VAG & 106 & 8 & 6.7 & 14.7 & 507 \\
HU-EtaKs VAG & 82 & 0 & 3.9 & 13.4 & 205 \\
HU-EtaK VAG & 82 & 0 & 4.0 & 13.9 & 203
\end{tabular}

Table 1: Number of the time steps $N_{\Delta t}$, number of the time step chops $N_{c h o p}$, average number of Newton iterations per time step $N_{\text {Newton }}$, average number of GMRES iterations per Newton step $N_{G M R e s}$ and CPU time for the three VAG schemes.

In conclusion, the positive HU VAG scheme provides similar solutions than the PPU version and exhibits an additional robustness in terms of nonlinear convergence for the simulation of highly heterogeneous media. Let us refer to [5] for more test-cases including large fracture networks. 

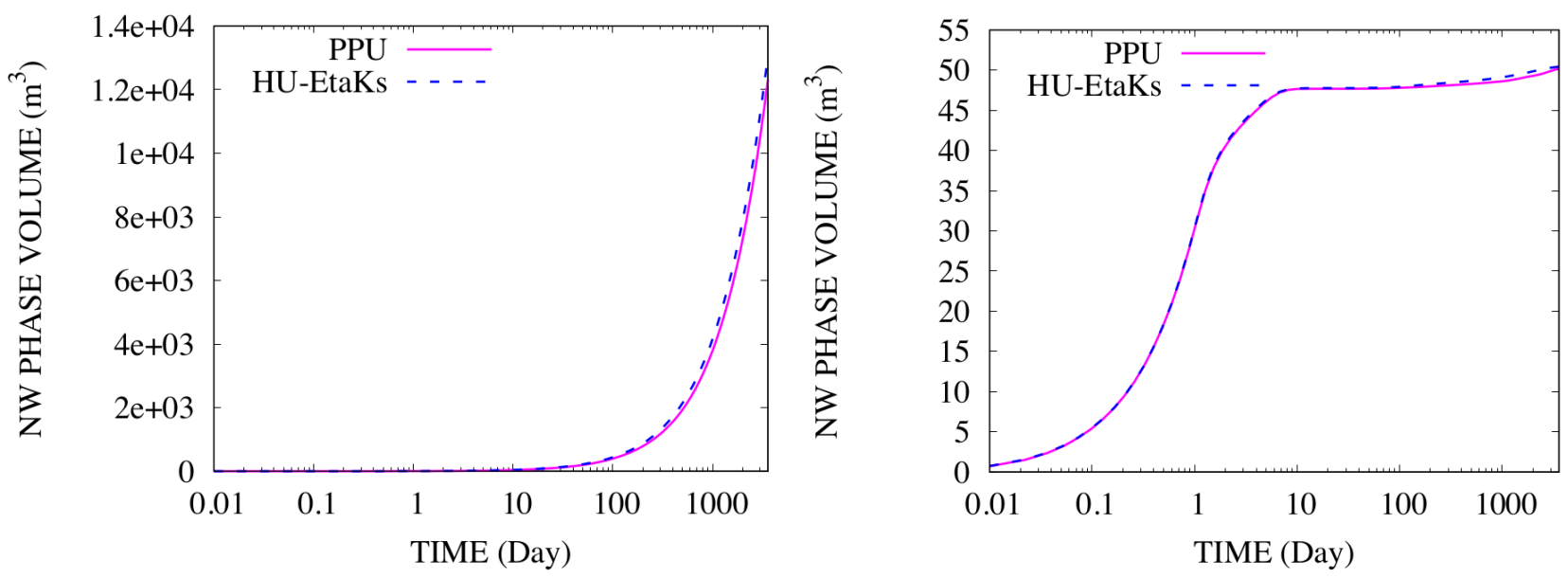

Figure 3: Oil saturation volumes in the matrix (left) and in the fracture network (right) as a function of time obtained for the PPU and HU-EtaKs VAG schemes.
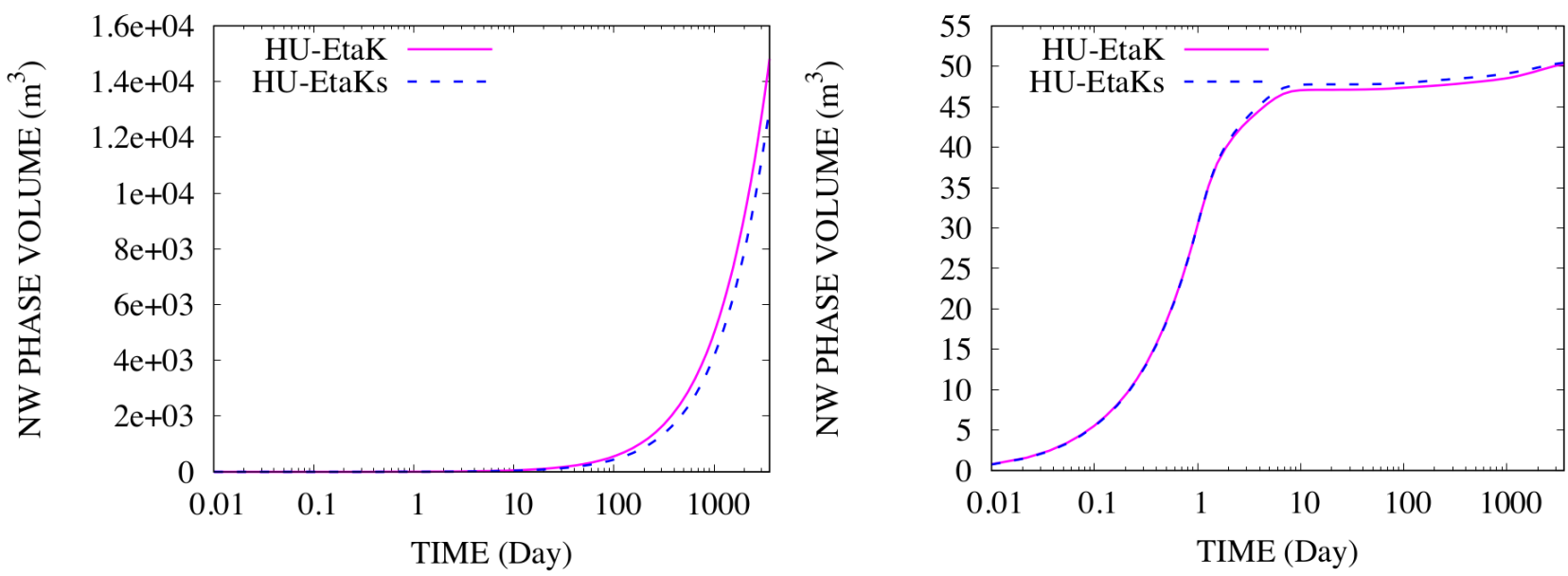

Figure 4: Oil saturation volumes in the matrix (left) and in the fracture network (right) as a function of time obtained for the HU VAG schemes with EtaKs and EtaK.

Acknowledgment : This work was supported by the French National Research Agency under the CHARMS ANR project ANR-16-CE06-0009.

\section{References}

[1] Andreianov, B., Brenner, K. and Cancès, C.: Approximating the vanishing capillarity limit of two-phase flow in multi-dimensional heterogeneous porous medium. ZAMM - J. of App. Math. and Mecha. / Zeit. für Ange. Math. und Mecha. 94 (7-8), 655-667 (2014)

[2] Brenier, Y., Jaffré, J.: Upstream differencing for multiphase flow in reservoir simulation. SIAM J. on Num. Ana. 28 (31), 685-696 (1991)

[3] Brenner, K., Groza, M., Jeannin, L., Masson, R., Pellerin, J.: Immiscible two-phase Darcy flow model accounting for vanishing and discontinuous capillary pressures: application to the flow in fractured porous media. Comp. Geoscie. 21 (5-6), 1075-1094 (2017) 

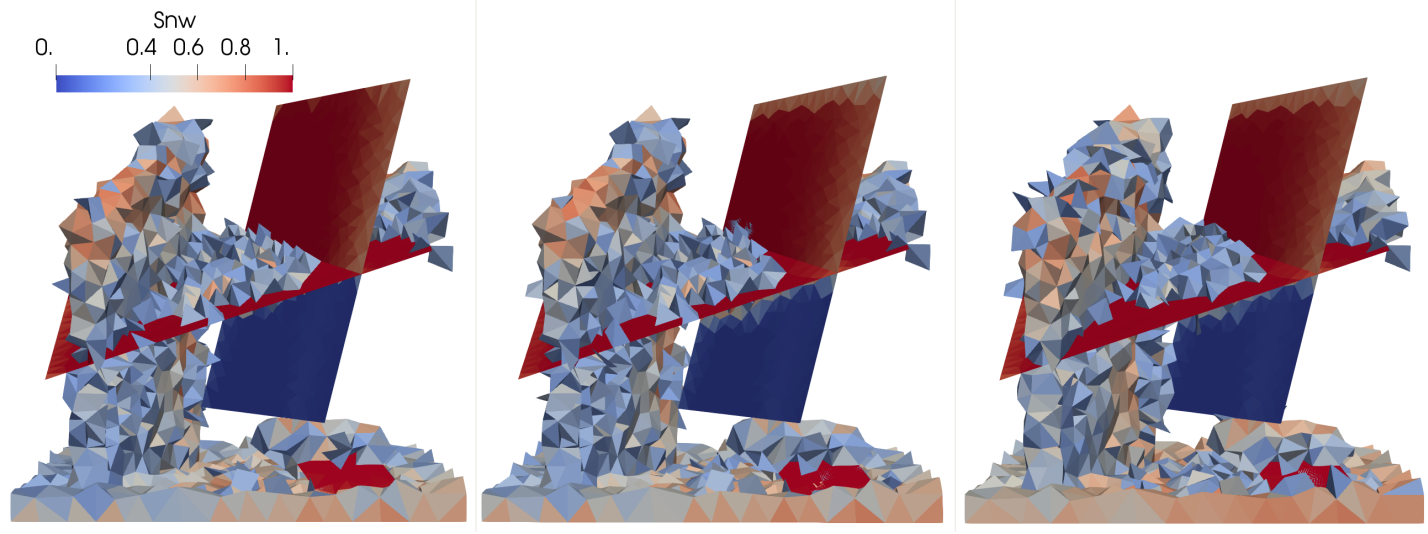

Figure 5: Oil saturation in the matrix for $s^{n w}>0.25$ and in the fractures at final time obtained for the PPU (left) and HU-EtaKs (middle) and HU-EtaK (right) VAG schemes.

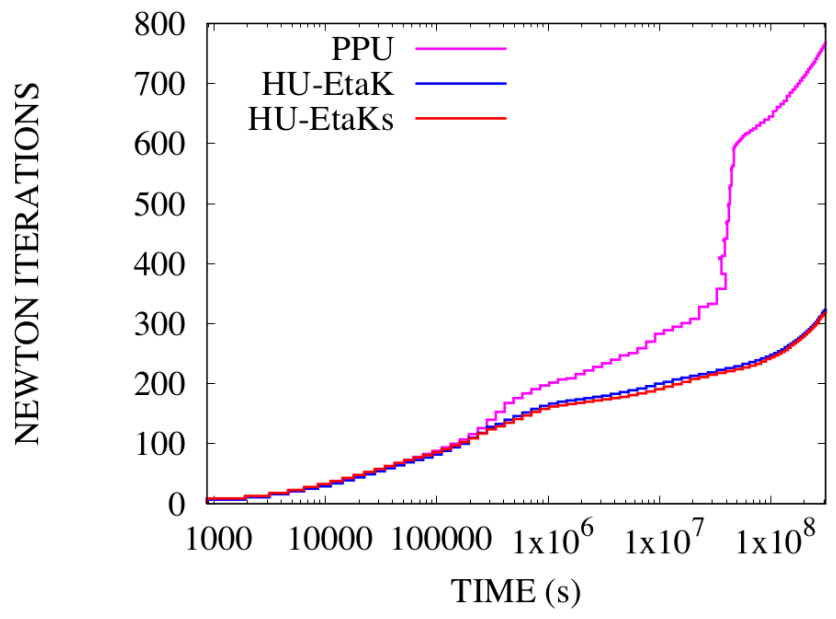

Figure 6: Total number of Newton iterations as a function of time for the three VAG schemes.

[4] Brenner, K., Masson, R.: Convergence of a vertex centred discretization of two-phase Darcy flows on general meshes. Inter. J. on Finite Volumes. 10, 1-37 (2013)

[5] Brenner, K., Masson, R., Quenjel, E.H.: Vertex approximate gradient discretization preserving positivity for two-phase Darcy flows in heterogeneous porous media. Preprint (2019)

[6] Hamon, F.P., Mallison, B.T., Tchelepi, H.A.: Implicit hybrid upwinding for two-phase flow in heterogeneous porous media with buoyancy and capillarity. Comp. Meth. in App. Mecha. and Engi. 331, 701-727 (2018)

[7] Quenjel, E.H.: Enhanced positive vertex-centered finite volume scheme for anisotropic convectiondiffusion equations. Accepted in ESAIM: Math. Mod. and Num. Ana. (2019) doi: 10.1051/m2an/2019075 